\title{
Degradation of Methylene Blue and Rhodamine B Using a New Visible Light-Responsive Photocatalyst, $\mathrm{KSb}_{2} \mathrm{PO}_{8-x} \mathrm{~N}_{y}$
}

\author{
${\text { G. } \text { Ravi }^{1} \cdot \text { CH. Sudhakar Reddy }}^{1} \cdot$ K. Sreenu ${ }^{1} \cdot$ Ravinder Guje $^{1} \cdot$ Radha Velchuri $^{1} \cdot$ M. Vithal $^{1}$
}

Received: 29 November 2015/Revised: 14 January 2016/Published online: 12 March 2016

(C) The Chinese Society for Metals and Springer-Verlag Berlin Heidelberg 2016

\begin{abstract}
Potassium phosphatoantimonates, $\mathrm{KSb}_{2} \mathrm{PO}_{8}(\mathrm{KSbPO})$ and $\mathrm{KSb}_{2} \mathrm{PO}_{8-x} \mathrm{~N}_{y}(\mathrm{KSbPON})$, are prepared by solidstate method. Urea is employed as a source of nitrogen for N-doping. Structural, morphological and optical properties of both KSbPO and KSbPON are characterized by powder X-ray diffraction (PXRD), scanning electron microscopy, energydispersive spectroscopy, Fourier transform infrared spectroscopy and UV-visible diffuse reflectance spectroscopy. Both samples are found to crystallize in a monoclinic lattice with Cc space group. The d-lines of KSbPO and KSbPON are refined by least square fitting the PXRD data, using POWD software to obtain their unit cell parameters. The bandgap energy $\left(E_{\mathrm{g}}\right)$ of both pristine and N-doped KSbPO is found from their Kubelka-Munk plot. The KSbPON/visible photocatalytic degradation of methylene blue and rhodamine $\mathrm{B}$ has been investigated in aqueous heterogeneous suspension. The generation of $\cdot \mathrm{OH}$ in the presence of $\mathrm{KSbPON}$ during the photoreaction is verified by a fluorescence technique using terephthalic acid (TA) as a probe.
\end{abstract}

KEY WORDS: Potassium phosphatoantimonates; Characterization; Organic dyes; Photodegradation

\section{Introduction}

Energy crisis and environmental pollution are the global problems, which have attracted the attention of all in general and researchers in particular. Upsetting levels of these issues demand increasingly stringent regulations that forced scientists to look for the more efficient techniques to solve the energy and environmental problems. Semiconductor-mediated photocatalysis, an emerging technique belonging to the class of advanced oxidation processes (AOPs), is known to be a right process to address the global concerns such as energy conservation and environmental

Available online at http://link.springer.com/journal/40195

M. Vithal

mugavithal@gmail.com

1 Department of Chemistry, University College of Science, Osmania University, Hyderabad 500007, India pollution $[1,2]$. The exploration of materials to identify effective photocatalysts for environmental purification and energy production has grown into an intense area of research in the past two decades. The study on semiconductor-based photosensitive materials for solar hydrogen production was initiated by the report of $\mathrm{TiO}_{2}$-based photoelectrochemical cell by Fujishima and Honda [3]. Subsequently, the significance of semiconductor photocatalysis for the degradation of harmful organic compounds was also recognized for environmental remediation [4-7]. It is noticed that most of the reported semiconductor materials are having bandgap energy $>3.0 \mathrm{eV}$ and exhibiting photocatalytic activity under UV irradiation only. In this context, different strategies have been followed to prepare photocatalysts with bandgap energy of $<3.0 \mathrm{eV}$ for the improvement of visible photon absorption $[8,9]$. One way of improving the visible light-driven photoactivity of a semiconductor is by reducing the bandgap energy through anion or cation substitution $[10,11]$. Substitution of oxygen by nitrogen/sulphur/fluorine in the lattice brings a change 
in the band structure due to the overlapping of $2 p$ orbitals of dopant with oxygen $2 p$ orbitals and leads to a reduction in the bandgap energy [12].

A substantial volume of work on photocatalysis relates to binary and ternary oxides only $[13,14]$. However, the materials other than oxides such as phosphates, borates and sulphates have been less investigated in the context of photocatalytic applications [15-18]. Particularly, alkali metal phosphatoantimonates have been the subject of intense interest due to their structural, ion-exchange and ionic conductivity properties [19]. Piffard et al. have reported a series of potassium phosphatoantimonates, K$\mathrm{O}-\mathrm{Sb}^{\mathrm{v}}-\mathrm{P}^{\mathrm{v}}$ [20-24]. The basic building units, $\mathrm{SbO}_{6}$ octahedra and $\mathrm{PO}_{4}$ tetrahedra, of these systems are associated in different ways giving rise to the one-dimensional (1D), two-dimensional (2D) or layer-type and three-dimensional (3D) tunnel-type network structures. Among these, layertype and tunnel-type phosphates are exhibiting good ionic conductivity, ion-exchange property and ability to accommodate radiative cations in the lattice [20, 22, 23]. However, photocatalytic applications of $\mathrm{K}-\mathrm{O}-\mathrm{Sb}^{\mathrm{v}}-\mathrm{P}^{\mathrm{v}}$ systems have not been exploited, despite their alluring properties which are more favourable for photocatalytic applications. Recently, we have reported the effect of cation/anion codoping on the photocatalytic performance of $\mathrm{Na}_{3}$ $\mathrm{SbO}\left(\mathrm{PO}_{4}\right)_{2}$, a phosphate in the Na-O-Sb-P system with layer structure [25]. In the present investigation, an attempt is made to substitute the anion $\left(\mathrm{O}^{2-}\right)$ partially by $\mathrm{N}^{3-}$, in one of the $\mathrm{K}-\mathrm{O}-\mathrm{Sb}^{\mathrm{v}}-\mathrm{P}^{\mathrm{v}}$ systems, $\mathrm{KSb}_{2} \mathrm{PO}_{8}$, and study their photocatalytic properties against the degradation of organic dyes.

\section{Experimental}

The parent material, $\mathrm{KSb}_{2} \mathrm{PO}_{8}(\mathrm{KSbPO})$, is prepared by solid-state reaction method as reported [24]. Analytical grade chemicals $\mathrm{KNO}_{3}, \mathrm{Sb}_{2} \mathrm{O}_{3}$ and $\mathrm{NH}_{4} \mathrm{H}_{2} \mathrm{PO}_{4}$ are used as starting materials. Stoichiometric amounts of all the reactants were weighed and ground in an agate mortar for one hour using spectral grade acetone followed by sequential heating in a muffle furnace. The samples are initially heated to $200{ }^{\circ} \mathrm{C}$ for $5 \mathrm{~h}$ to enable the decomposition of ammonium dihydrogen phosphate and potassium nitrate. The resultant powder is ground for one hour and sintered in air at $920{ }^{\circ} \mathrm{C}$ for $12 \mathrm{~h}$ for densification.

The N-doped $\mathrm{KSb}_{2} \mathrm{PO}_{8}$ is obtained by heating a mixture of KSbPO and urea at $400{ }^{\circ} \mathrm{C}$ for $2 \mathrm{~h}$ in a muffle furnace in air. The weight ratio of KSbPO to urea is 1:4. The resultant powder is washed several times with deionized water to remove excess unreacted urea. The colour of the N-doped $\mathrm{KSbPO}$ is found to be light yellow and designated as KSbPON.

\subsection{Characterization}

The room-temperature $\mathrm{X}$-ray diffractograms are recorded using a Rigaku Miniflex powder X-ray diffractometer $(\mathrm{CuK} \alpha, \lambda=0.15406 \mathrm{~nm})$ in the $2 \theta$ range $10^{\circ}-80^{\circ}$ for phase confirmation. The unit cell parameters of KSbPO and KSbPON are refined by least square fitting the powder $\mathrm{X}$-ray diffraction (XRD) data, using POWD software. JASCO V650 UV-Vis spectrophotometer is used for UVVis diffuse reflectance spectral (DRS) measurements in the range $200-800 \mathrm{~nm} . \mathrm{BaSO}_{4}$ is used as the reflectance standard. Scanning electron microscopy (SEM) images of all the materials are recorded on a HITACHI SU-1500 variable pressure scanning electron microscope (VP-SEM). Oxygen-nitrogen-hydrogen $(\mathrm{O}-\mathrm{N}-\mathrm{H})$ analysis is carried out using an LECO O-N-H 836 analyser. Infrared spectra are recorded in the form of $\mathrm{KBr}$ pellets in the wave number range of $1600-400 \mathrm{~cm}^{-1}$ using a JASCO IR-5300 spectrometer.

\subsection{Photocatalytic Experiments}

\subsubsection{Photodegradation of Methylene Blue (MB) and Rhodamine $B(R h B)$}

The photocatalytic activities of KSbPO and KSbPON samples are evaluated by photodegradation of methylene blue $(\mathrm{MB})$ and rhodamine $\mathrm{B}(\mathrm{RhB})$ under visible light irradiation using HEBER visible annular type photoreactor equipped with a $300-\mathrm{W}$ tungsten lamp (wavelength range $380-840 \mathrm{~nm}$ ). In a typical process, $50 \mathrm{~mL}$ of aqueous MB solution with an initial concentration of $10 \mathrm{mg} \mathrm{L}^{-1}$ and $50 \mathrm{mg}$ of catalyst are taken in a cylindrical-shaped glass reactor at room temperature in air. The suspension is stirred in the dark for 60 min to establish adsorption-desorption equilibrium before irradiation. At regular time intervals of $30 \mathrm{~min}$, about $2-3 \mathrm{~mL}$ of the solution is collected and centrifuged to remove the catalyst particles. The change in the concentration of $\mathrm{MB}$ is obtained by recording the absorbance at $664 \mathrm{~nm}$ using JASCO V650 UV-Vis spectrophotometer. The amount of degradation is reported as $\mathrm{Cl}$ $C_{\text {eq }}$, where $C$ is the concentration of MB at each irradiated time, and $C_{\mathrm{eq}}$ is the concentration of $\mathrm{MB}$ when the adsorption-desorption equilibrium is achieved. The similar procedure is adopted for degradation of rhodamine $\mathrm{B}$ $(\mathrm{RhB})$. The concentration and the $\lambda_{\max }$ of $\mathrm{RhB}$ are $1 \times 10^{-5} \mathrm{~mol} \mathrm{~L}^{-1}$ and $554 \mathrm{~nm}$, respectively.

\subsubsection{Mechanistic Studies of Photocatalyst}

The formation of $\cdot \mathrm{OH}$ radicals during photocatalysis is followed by the measurement of fluorescence intensity as reported [26]. Typically, $50 \mathrm{mg}$ of catalyst is suspended in 
$50 \mathrm{~mL}$ of a $0.02 \mathrm{~mol} \mathrm{~L}^{-1} \mathrm{NaOH}$ solution containing $3 \mathrm{mmol} \mathrm{L}^{-1}$ terephthalic acid (TA). The suspension is stirred in the dark for $60 \mathrm{~min}$ before visible light $(300 \mathrm{~W}$ tungsten lamp) illumination. Then, $2-3 \mathrm{~mL}$ of the suspension is taken out at 60 -min intervals, filtered and recorded fluorescence spectra using Shimadzu RF-5301PC fluorescence spectrophotometer. The photogenerated $\cdot \mathrm{OH}$ radicals react with TA to form the 2-hydroxy terephthalic acid (TAOH), which shows a characteristic fluorescence band centred at $425 \mathrm{~nm}$. The increase in the fluorescence intensity of TAOH is directly proportional to the concentration of photogenerated $\cdot \mathrm{OH}$ radicals. The excitation wavelength is fixed at $320 \mathrm{~nm}$.

\section{Results and Discussion}

The parent material, $\mathrm{KSb}_{2} \mathrm{PO}_{8}(\mathrm{KSbPO})$, is prepared by solidstate reaction at $920{ }^{\circ} \mathrm{C} / 12 \mathrm{~h}$. The structure of $\mathrm{KSb}_{2} \mathrm{PO}_{8}$ can be described as the linkage of $\mathrm{PO}_{4}$ tetrahedra with $\mathrm{SbO}_{6}$ octahedra sharing vertices or edges. The $\mathrm{SbO}_{6}$ octahedra share both corners and edges and are linked to the $\mathrm{PO}_{4}$ tetrahedral via corners as shown in Fig. 1. Each tetrahedral $\mathrm{PO}_{4}$ unit is connected to four $\mathrm{SbO}_{6}$ octahedra units. It was reported that $\mathrm{K}^{+}$ions present in this structure cannot be exchanged with the other cations due to its highly closepacked structure [20-24]. Our attempts to exchange $\mathrm{K}^{+}$with other mono- or di-valent ions were also unsuccessful. As mentioned earlier, the bandgap energy can also be modulated by anion doping. So, in the present investigation, an attempt is made to substitute $\mathrm{N}^{3-}$ into KSbPO lattice. A solid-state method is employed using urea as a nitrogen source. It is well known that during the nitrogen doping by ceramic method (at $\left.400{ }^{\circ} \mathrm{C}\right)$ there is a possibility of (1) carbon nitrogen $(\mathrm{CN})$ based polymeric products entering into lattice [27, 28], and (2) deposition of nitrites and hyponitrites on the surface [29]. These nitrogen-based products can be detected by powder XRD and IR techniques.
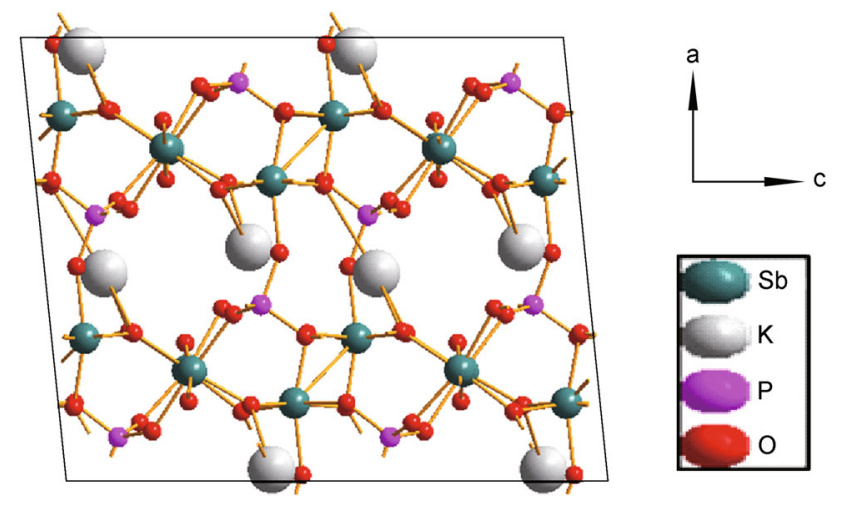

Fig. 1 Schematic structure of $\mathrm{KSb}_{2} \mathrm{PO}_{8}$
The powder XRD patterns of KSbPO and KSbPON are recorded for phase conformation (Fig. 2a). The observed XRD patterns of KSbPO show d-lines, which are consistent with reported $\mathrm{KSb}_{2} \mathrm{PO}_{8}$ data [JCPDF: 78-0492]. Li et al. [29] have reported that the powder XRD patterns of CN polymer-sensitized $\mathrm{N}$-doped oxides are often characterized by a broad peak in the $2 \theta$ region $27^{\circ}-32^{\circ}$ and disappearance of majority of the parent (undoped) phospho-oxide diffraction peaks. However, in the present investigation, the powder XRD pattern of KSbPON is identical to that of $\mathrm{KSbPO}$ without any loss in the number of diffraction peaks, and no additional peak(s) is/are observed in the $2 \theta$ region of $27^{\circ}-32^{\circ}$. To authenticate the incorporation of nitrogen into the KSbPO lattice, the powder patterns of $\mathrm{KSbPO}$ and $\mathrm{KSbPON}$ are plotted on an expanded scale. The diffraction peaks of KSbPON show a systematic shift towards lower $2 \theta$ value compared to that of $\mathrm{KSbPO}$ (Fig. 2b). These observations indicate the substitution of nitrogen into the KSbPO lattice. The unit cell parameters of $\mathrm{KSbPO}$ and KSbPON are refined by least square fitting the powder data, using POWD software to obtain their unit cell parameters. Both samples are found to crystallize in a monoclinic lattice with $\mathrm{Cc}$ space group. The observed and calculated $d$-values along with their $h k l$ values for the $\mathrm{KSbPO}$ and KSbPON are given in Table 1. The obtained unit cell parameters are listed in Table 2 . The small variations in unit cell lengths and unit cell volume of KSbPON are due to the partial replacement of oxygen by nitrogen in $\mathrm{SbO}_{6}$ octahedra and/or $\mathrm{PO}_{4}$ tetrahedra of KSbPO. Since the ionic radius of $\mathrm{N}^{3-}(0.171 \mathrm{~nm})$ is greater than that of $\mathrm{O}^{2-}$ $(0.140 \mathrm{~nm})$, partial substitution of $\mathrm{O}$ by $\mathrm{N}$ should increase the unit cell parameters. The average crystallite size of both powders was calculated from the Scherrer's formula [30]

$t=\frac{0.9 \lambda}{\beta \cos \theta}$,

where $t$ is the thickness in angstrom $(\AA)$ and corresponds to the crystallite diameter assuming a spherical shape; $\lambda$ is the wavelength of the $\mathrm{X}$-ray used; $\theta$ is the Bragg angle and $\beta$ is the full width at half maximum measured in radians of d-line in the powder XRD pattern. The crystallite sizes of the KSbPO and KSbPON were found to be 55 and $49 \mathrm{~nm}$, respectively.

FTIR spectra of KSbPO and KSbPON are shown in Fig. 3. The vibrational bands observed in FTIR spectrum of KSbPO are similar to the reported data [31]. The vibrational bands observed for N-doped KSbPO vary significantly from the parent KSbPO in two ways. First, the band positions in the $400-1250 \mathrm{~cm}^{-1}$ region have been shifted towards lower wavenumbers. Second, the new bands in the region $1250-1600 \mathrm{~cm}^{-1}$ have appeared. The shift of KSbPON vibrational bands towards lower wavenumber also suggests that some oxygen atoms in KSbPO lattice are 

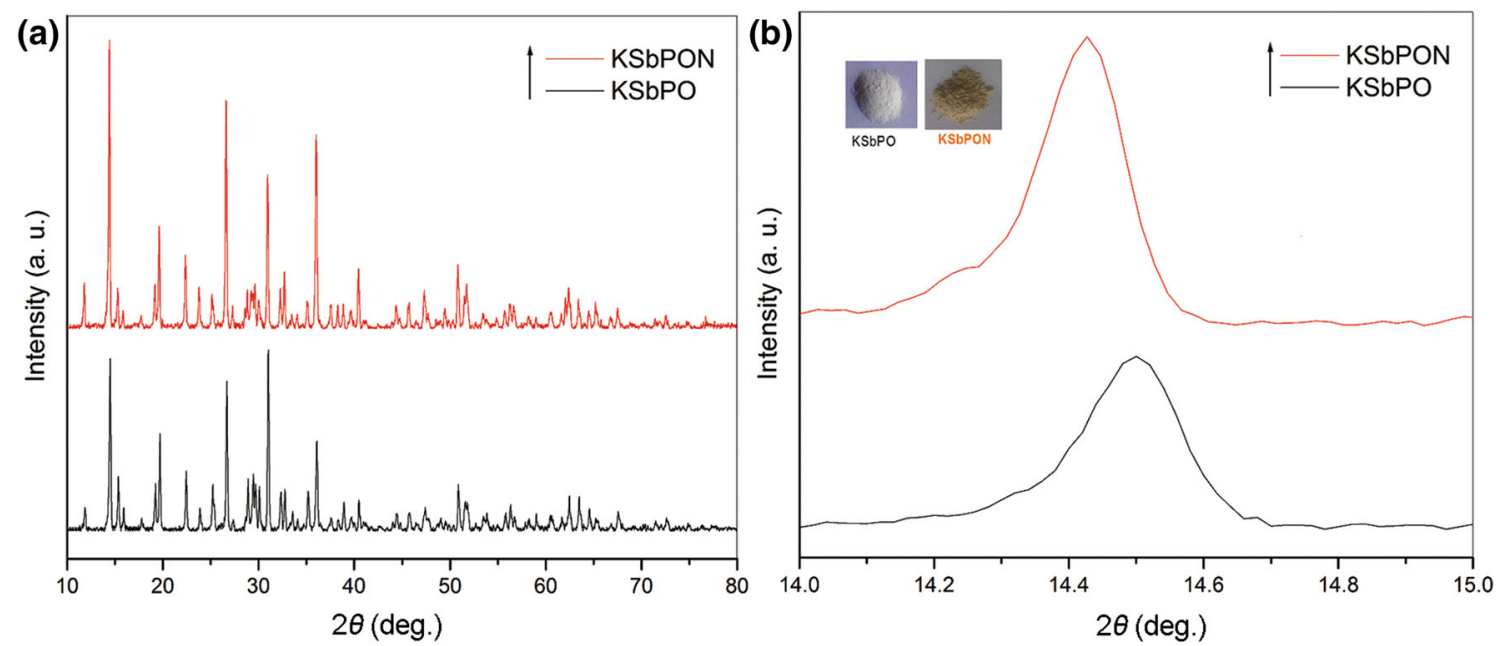

Fig. 2 XRD patterns of a KSbPO and KSbPON, b expanded XRD patterns of KSbPO and KSbPON. Insets show the colour of the samples

Table 1 Observed and calculated $d$-values along with their $h k l$ values for the KSbPO and KSbPON

\begin{tabular}{|c|c|c|c|c|}
\hline \multirow[t]{2}{*}{$h k l$} & \multicolumn{2}{|l|}{ KSbPO } & \multicolumn{2}{|c|}{ KSbPON } \\
\hline & $d_{\mathrm{obs}}$ & $d_{\mathrm{cal}}$ & $d_{\mathrm{obs}}$ & $d_{\mathrm{cal}}$ \\
\hline 002 & 7.4637 & 7.4634 & 7.4715 & 7.4737 \\
\hline 110 & 6.1258 & 6.1257 & 6.1319 & 6.1334 \\
\hline 200 & & 6.1151 & & 6.1259 \\
\hline-1111 & 5.7711 & 5.7712 & 5.7752 & 5.7775 \\
\hline 111 & 5.5684 & 5.5685 & 5.5705 & 5.5764 \\
\hline-202 & 4.9851 & 4.9849 & 4.9906 & 4.9906 \\
\hline 112 & 4.6211 & 4.6212 & 4.6223 & 4.6281 \\
\hline 202 & 4.5117 & 4.5109 & 4.5117 & 4.5198 \\
\hline-113 & 3.9621 & 3.9620 & 3.9645 & 3.9664 \\
\hline 113 & 3.7698 & 3.7694 & 3.7738 & 3.7752 \\
\hline 020 & 3.5387 & 3.5388 & 3.5444 & 3.5425 \\
\hline-204 & 3.3400 & 3.3399 & 3.3426 & 3.3434 \\
\hline-114 & 3.2616 & 3.2615 & 3.2716 & 3.2712 \\
\hline 114 & 3.1172 & 3.1173 & 3.1251 & 3.1220 \\
\hline 312 & 3.0895 & 3.0896 & 3.0937 & 3.0952 \\
\hline 204 & 3.0576 & 3.0575 & 3.0583 & 3.0562 \\
\hline-221 & 3.0309 & 3.0309 & 3.0351 & 3.0344 \\
\hline 221 & 2.9706 & 2.9707 & 2.9902 & 2.9895 \\
\hline
\end{tabular}

substituted by nitrogen atoms. When $\mathrm{Sb}-\mathrm{O}(\mathrm{P}-\mathrm{O})$ bonds are replaced by $\mathrm{Sb}-\mathrm{N}(\mathrm{P}-\mathrm{N})$, the increase in bond length of $\mathrm{Sb}-\mathrm{N}(\mathrm{P}-\mathrm{N})$ is observed (due to a higher ionic radius of $\mathrm{N}^{3-}$ ), and this leads to a decrease in the force constant [32]. Lower force constant results in a lower stretching or bending vibrational frequency. Thus, the observed band positions are consistent with substitution of nitrogen into the lattice. The new bands around 1260, 1330, 1425 and $1490 \mathrm{~cm}^{-1}$ are attributed to the presence of interstitial $\mathrm{N}-\mathrm{O}$ species in the form of hyponitrite and nitrite on the surface of KSbPON [29]. The vibration bands observed for KSbPO and KSbPON are listed in Table 3. The presence of water in $\mathrm{KSbPON}$ is further supported by thermogravimetric analysis (TGA). Thermogravimetric (TG) curve of the $\mathrm{KSbPON}$ is shown in Fig. 4. This curve is characterized by two weight loss regions. In the temperature region $30-300{ }^{\circ} \mathrm{C}$, the weight loss is $1.8 \%$. This weight loss may be attributed to the adsorbed and crystalline water of the lattice. The observed weight loss $(1 \%)$ in $300-500{ }^{\circ} \mathrm{C}$ region indicates the decomposition of chemisorbed unstable species such as hyponitrite and nitrite.

The morphological characterization of $\mathrm{KSbPO}$ and KSbPON is carried out using scanning electron microscopy (SEM). The SEM images of both the samples are obtained

Table 2 Unit cell parameters and volumes of KSbPO and KSbPON

\begin{tabular}{llllll}
\hline Samples & $a(\AA) \pm(0.005)$ & $B(\AA) \pm(0.005)$ & $c(\AA) \pm(0.005)$ & $\beta\left({ }^{\circ}\right)$ & $\mathrm{Vol}^{\circ}(\AA)^{3}$ \\
\hline KSbPO & 12.29 & 7.078 & 15.00 & 95.83 & 1298.78 \\
KSbPON & 12.31 & 7.08 & 15.02 & 95.78 \\
\hline
\end{tabular}



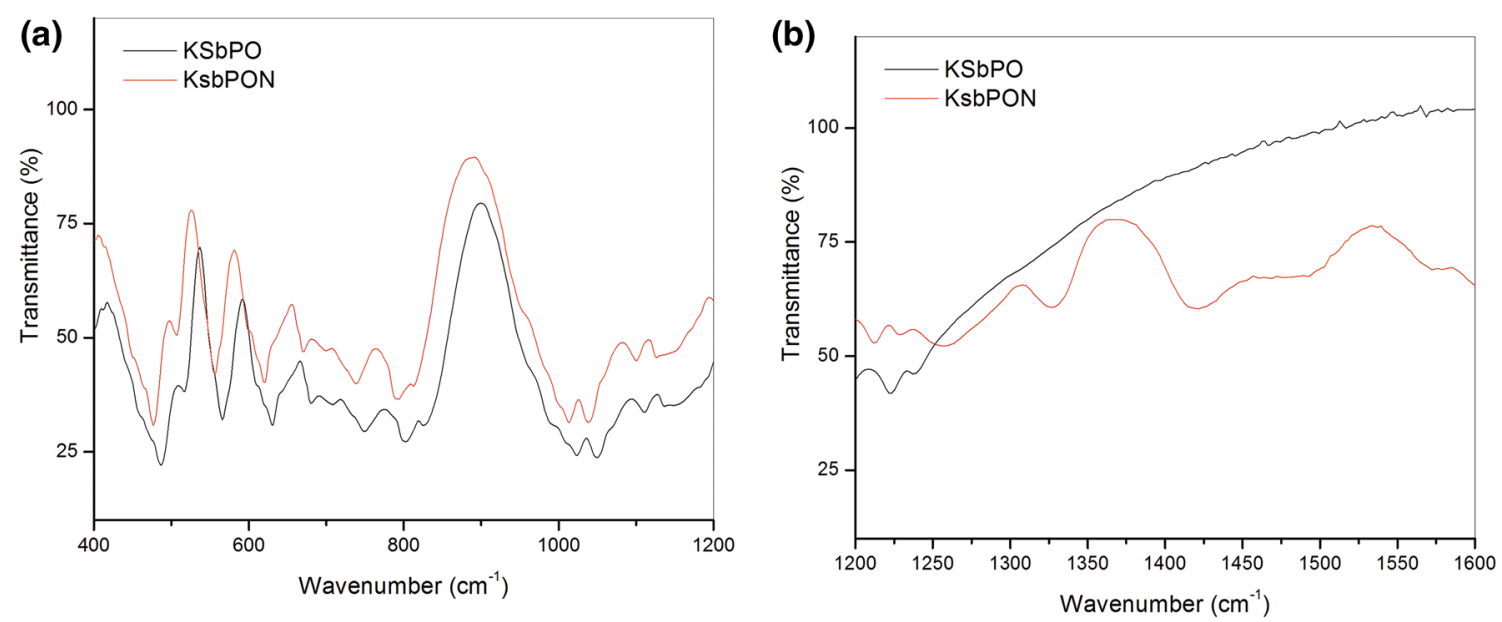

Fig. 3 FTIR spectra of KSbPO and KSbPON in the regions a $400-1200 \mathrm{~cm}^{-1}$, b $1200-1600 \mathrm{~cm}^{-1}$

Table 3 Vibration bands observed for KSbPO and KSbPON $\left(\mathrm{cm}^{-1}\right)$

\begin{tabular}{lc}
\hline KSbPO & KSbPON \\
\hline 487 & 473 \\
517 & 503 \\
567 & 551 \\
628 & 617 \\
677 & 665 \\
744 & 734 \\
799 & 787 \\
821 & 814 \\
1021 & 1009 \\
1051 & 1038 \\
1106 & 1098 \\
1134 & 1123 \\
1221 & 1207 \\
- & 1260 \\
- & 1330 \\
- & 1425 \\
- & 1490 \\
\hline
\end{tabular}

at different magnifications $(1,10$ and $20 \mu \mathrm{m})$. As shown in Fig. 5, it is observed that KSbPO microcrystals consist of the irregularly shaped plate-like particles. The KSbPON particles appear as rod shape with significant agglomeration. Further, the substitution of $\mathrm{N}^{3-}$ ions into KSbPO lattice is verified by recording their energy-dispersive spectra (EDS). The EDS profiles of both the samples are also shown in Fig. 5. The weight ratio of $K, S b$ and $P$ obtained from EDS data for KSbPO was noticed to be close to expected values (i.e. $\mathrm{K}: \mathrm{Sb}: \mathrm{P}=1: 2: 1$ ) confirming the molecular formula as $\mathrm{KSb}_{2} \mathrm{PO}_{8}$. The presence of nitrogen

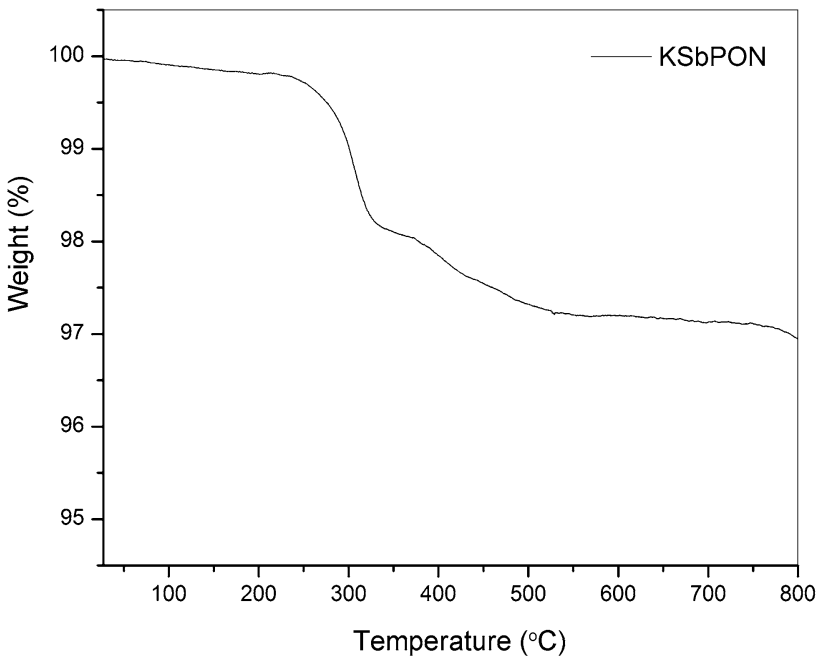

Fig. 4 Thermogravimetric profile of KSbPON

in EDS of KSbPON indicates its substitution into KSbPO lattice, and the molecular formula of KSbPON can be written as $\mathrm{KSb}_{2} \mathrm{PO}_{8-x} \mathrm{~N}_{y}$. The weight percentage of nitrogen is measured quantitatively by $\mathrm{O}-\mathrm{N}-\mathrm{H}$ analysis. The weight percentage of nitrogen is found to be 22.08 .

$\mathrm{UV}-\mathrm{Vis}$ diffuse reflectance spectroscopy is used to study the optical properties of the KSbPO on nitrogen doping. Figure 6a, b shows the UV-Vis absorption and Kubelka-Munk (KM) plot of KSbPO and KSbPON, respectively. The absorption spectra show that the KSbPON has higher light absorption intensity and redshifted absorption edge compared to the KSbPO is about $85 \mathrm{~nm}$. The change in colour of KSbPO from white to light yellow upon the nitrogen doping also endorsed the red shift of the absorption edge (Fig. 2b, inset). The bandgap energy of both the samples was estimated from a KM plot $\left[(K h v)^{1 /}\right.$ ${ }^{2}$ versus $h v$, where $K=\frac{(1-R)^{2}}{R}, R$ is reflectance (\%)] and $h v$ 

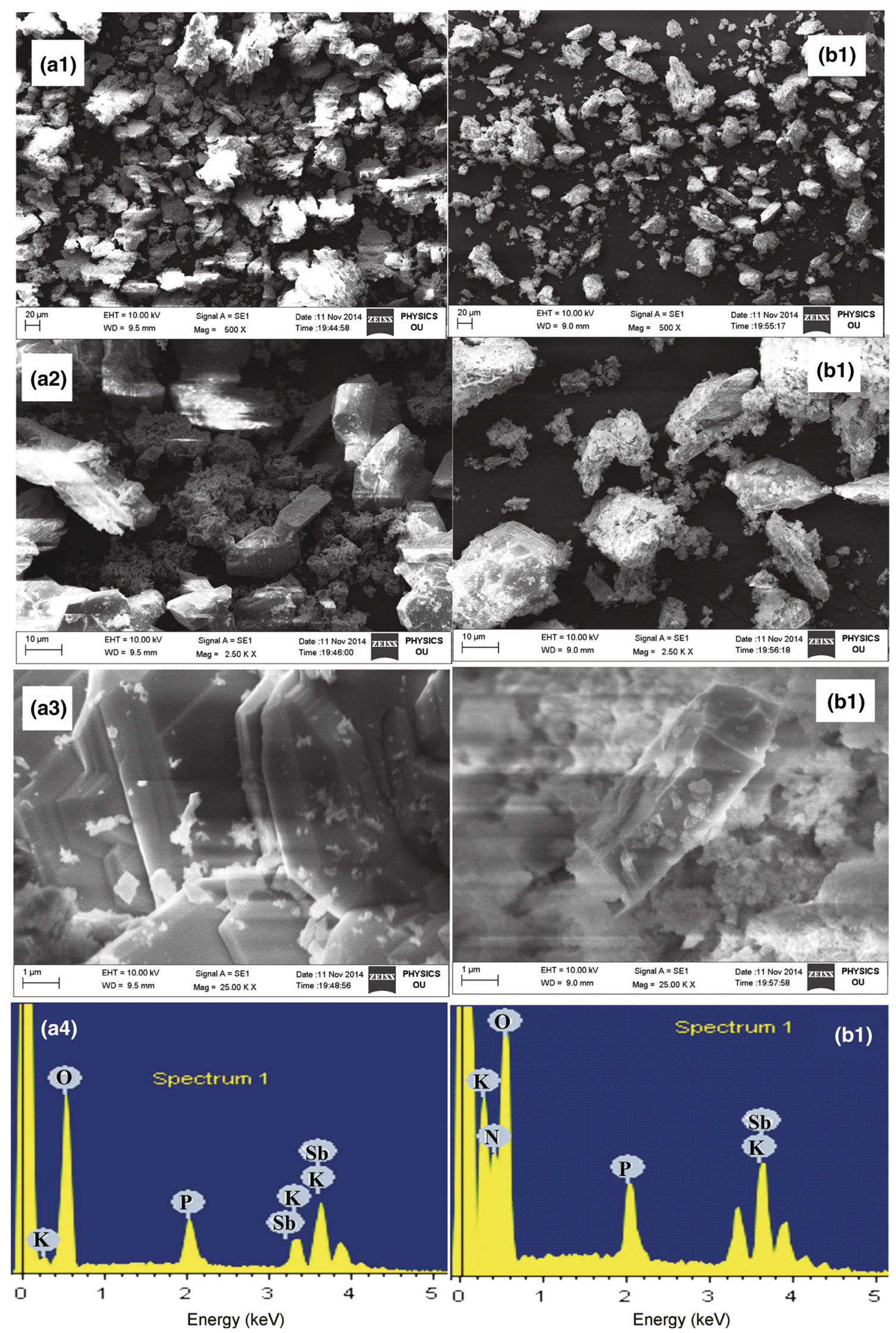

Fig. 5 SEM-EDS of a1-a4 KSbPO and b1-b4 KSbPON 
is the energy of absorbed light). The optical bandgaps of the $\mathrm{KSbPO}$ and KSbPON are 3.35 and $2.72 \mathrm{eV}$, respectively. This shows that the nitrogen doping acted as a bandgap modifier due to the contribution of its $2 p$ orbitals to the valence band consisting of oxygen $2 p$ orbitals [33].

The photocatalytic activities of KSbPO and KSbPON are evaluated by the degradation of $\mathrm{MB}$ and $\mathrm{RhB}$ under visible light irradiation. It is observed that the KSbPO has not shown any activity towards $\mathrm{MB}$ and $\mathrm{RhB}$ degradation (Fig. 7). This may be due to its higher bandgap energy $(3.35 \mathrm{eV})$, and in the present experimental set-up, the light (tungsten lamp) energy is much lower than $3.35 \mathrm{eV}$. On the other hand, KSbPON has shown activity against both MB and $\mathrm{RhB}$ degradation. Figure $8 \mathrm{a}$ shows the temporal changes in the concentration of $\mathrm{MB}$ and $\mathrm{RhB}$ organic dyes in the presence of $\mathrm{KSbPON}$ catalyst upon visible light illumination. The degradation of $\mathrm{MB} / \mathrm{RhB}$ is measured by following the variations in maximum absorption in their UV-visible spectra at $664 / 554 \mathrm{~nm}$. Before the irradiation, the KSbPON material is kept in the dark chamber to attain the adsorption-desorption equilibrium. In the presence of light illumination, it is noticed that the degradation of $\mathrm{MB} /$ $\mathrm{RhB}$ is increased with an increase in the irradiation time (Fig. 8a). The extents of MB and RhB degradation after 180 min of irradiation observed for the KSbPON are $82 \%$ and $58 \%$, respectively.

The kinetics of $\mathrm{MB}$ and $\mathrm{RhB}$ degradation in the presence of KSbPON is also studied. The degradation reaction of $\mathrm{MB} / \mathrm{RhB}$ with KSbPON shows pseudo-first-order kinetics (Langmuir-Hinshelwood model) [34]. The results are nearly steady with linear equation

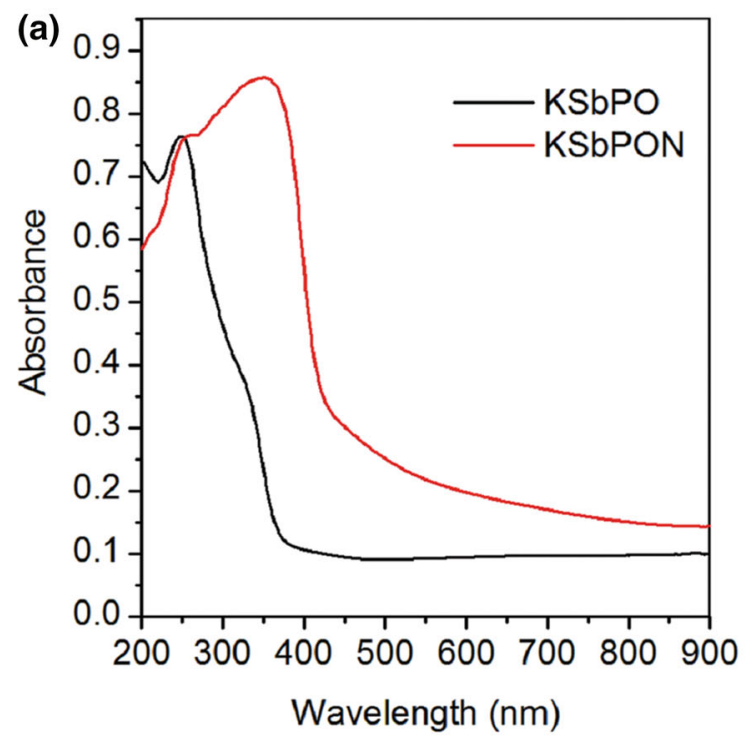

Fig. 6 UV-Vis a absorption and b EDS profiles of KSbPO and KSbPON

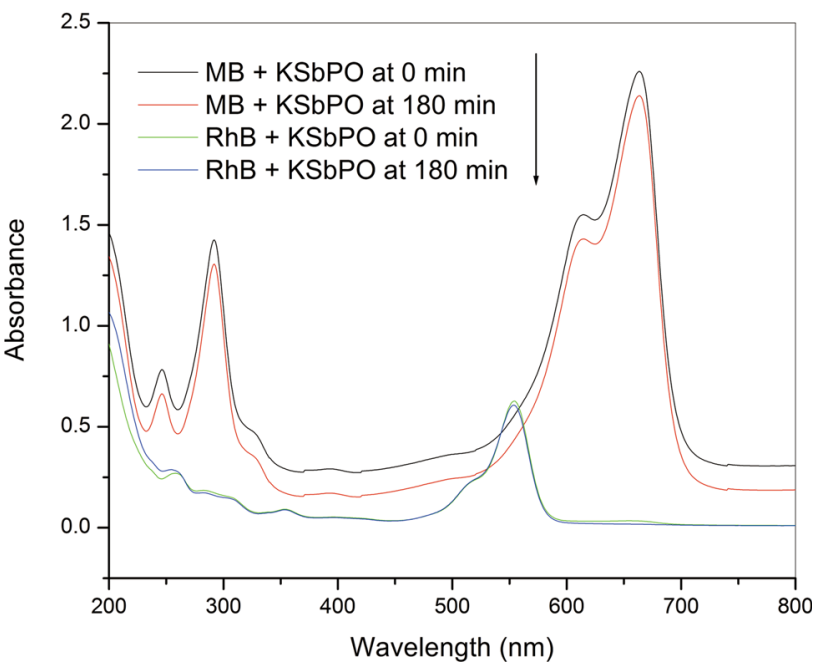

Fig. $7 \mathrm{UV}-\mathrm{Vis}$ absorption spectra of $\mathrm{MB}$ and $\mathrm{RhB}$ dye at 0 and 180 min under visible light irradiation in the presence of $\mathrm{KSbPO}$

$\ln \left(C / C_{0}\right)=-k t$

For the pseudo-first-order reaction, the half-life time $\left(t_{1 / 2}\right)$ can be determined according to the equation

$t_{1 / 2}=\frac{\ln 2}{k}$.

Figure 8b represents a linear correlation $\left(R^{2}\right)$, suggesting that the degradation reaction follows the first-order kinetics. The slope of the linear line gives the first-order rate constant. The kinetic parameters $\left(k, t_{1 / 2}\right)$ for both photocatalysts are given in Table 4.

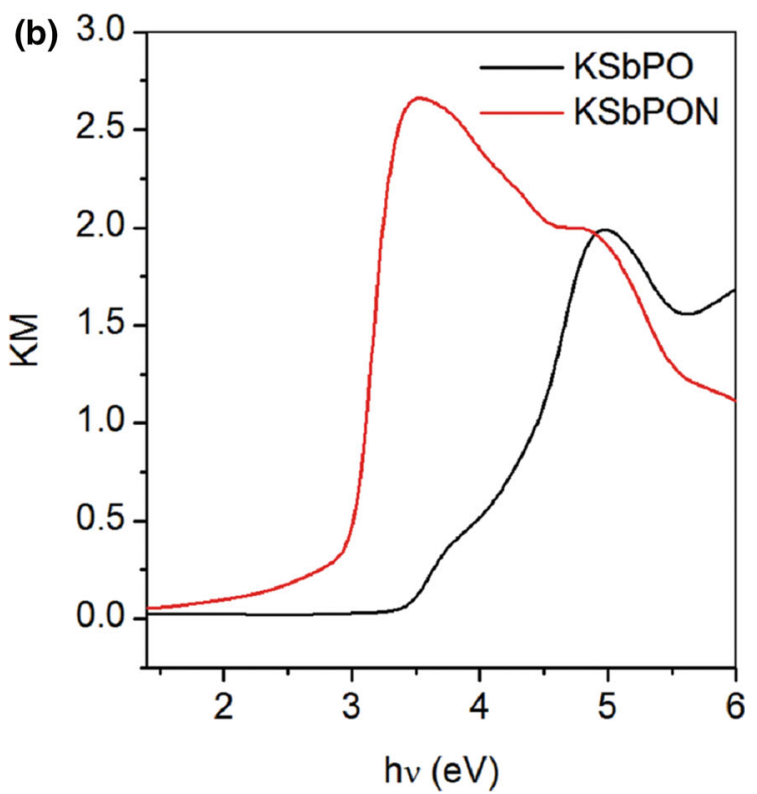



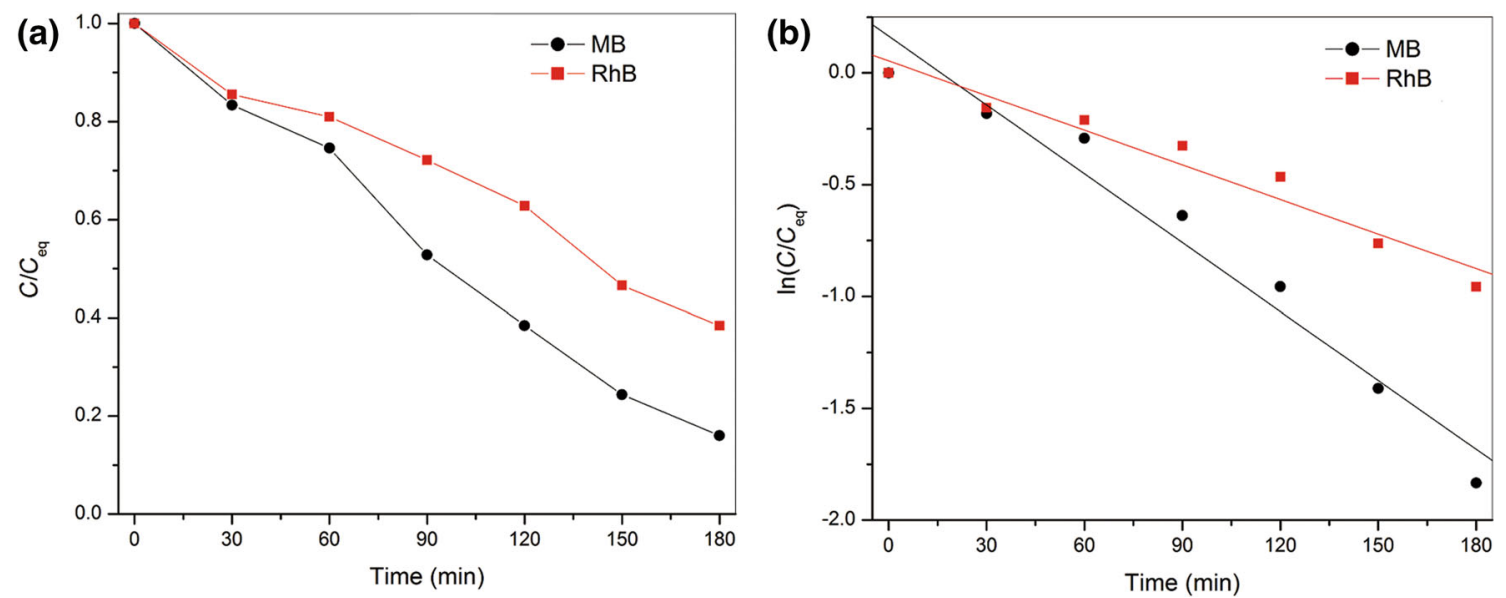

Fig. 8 a Variation in concentration and $\mathbf{b}$ first-order kinetics plots of MB and RhB with irradiation time against KSbPON

Table 4 Kinetic parameters, rate constant $(k)$ and half-life time $\left(t_{1 / 2}\right)$ of KSbPON in the first-order photocatalytic reaction

\begin{tabular}{lllr}
\hline Photocatalyst (dye) & First-order rate constant, $k\left(\mathrm{~min}^{-1}\right)$ & Square of correlation coefficient $R^{2}$ & $t_{1 / 2}\left(\mathrm{~min}^{-1}\right)$ \\
\hline KSbPON (MB) & $0.0102 \pm 0.0009$ & 0.962 & 68 \\
KSbPON (RhB) & $0.0051 \pm 0.0005$ & 0.952 & 136 \\
\hline
\end{tabular}

The degradation of organic pollutants on the surface of a catalyst is principally initiated by illumination of light of energy equal to or greater than the bandgap energy of catalyst, resulting in the promotion of the valence band electron into its conduction band. The formation of an electron (in the conduction band) and hole (in the valence band) is the first step in the mechanism of photocatalysis. These photoinduced electron and hole could migrate to the surface to react with the adsorbed reactants in the desired process, or undergo undesired recombination. Therefore, the generation and separation of photoinduced electronhole pairs are the key factors in a photocatalytic reaction. They can involve in various oxidation and reduction reactions with adsorbed species such as $\mathrm{H}_{2} \mathrm{O}$ and $\mathrm{O}_{2}$, producing hydroxyl $(\cdot \mathrm{OH})$ and superoxide radicals, respectively. These radicals react with the dye molecules and degrade them into harmless inorganic molecules such as $\mathrm{CO}_{2}$ and $\mathrm{H}_{2} \mathrm{O}$.

Predominantly, the degradation rate of organic dyes is directly proportional to the possibility of growth of $\cdot \mathrm{OH}$ on the catalysts surface and their reaction with dye molecules. In the present investigation, the generation of $\cdot \mathrm{OH}$ in the presence of KSbPON during the photoreaction is verified by a fluorescence technique using TA as a probe. The $\cdot \mathrm{OH}$ species react with TA to give 2-hydroxy terephthalic acid $(\mathrm{TAOH})$ which has a characteristic fluorescence signal at $426 \mathrm{~nm}$. Therefore, the formation of TAOH as a result of photocatalytic hydroxylation of TA in the presence of

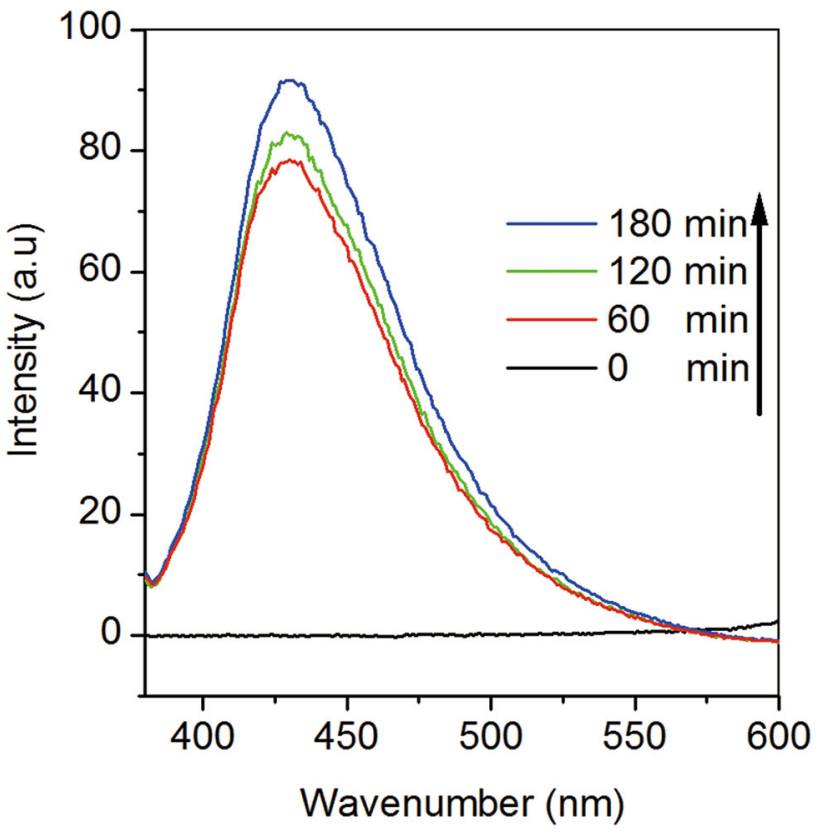

Fig. 9 Fluorescence spectra of visible light-irradiated KSbPON suspensions in $3 \mathrm{mmol} \mathrm{L}^{-1}$ terephthalic acid $\left(\lambda_{\text {excitation }}=320 \mathrm{~nm}\right)$

$\mathrm{KSbPON}$ is used as a sign for the $\cdot \mathrm{OH}$ generation. It is noticed from Fig. 9 that the fluorescence intensity at $426 \mathrm{~nm}$ of TA solution in the presence of KSbPON is found to increase with the rise in visible light irradiation time. Thus, the escalation in fluorescence intensity with the 
rise in visible light irradiation time for $\mathrm{KSbPON}$ catalyst indicates generation of $\cdot \mathrm{OH}$ (or formation of TAOH) [35].

\section{Conclusions}

Nitrogen-doped KSbPO is prepared by a solid-state method using urea as a source of nitrogen. KSbPON is crystallized in a monoclinic lattice. The nitrogen doping into KSbPO lattice is confirmed from (a) shift in the d-lines of powder patterns, (b) the presence of $\mathrm{N}$ peak in its EDS profiles, (c) change in the colour of the samples, (d) red shift in the absorption edge in UV-visible absorption spectra and (e) the presence of new IR bands in KSbPON. The bandgap energy of $\mathrm{KSbPO}$ is reduced about $0.63 \mathrm{eV}$ over the nitrogen doping. The extents of $\mathrm{MB}$ and $\mathrm{RhB}$ degradation after $180 \mathrm{~min}$ of irradiation observed for the KSbPON are $82 \%$ and $58 \%$, respectively. The photodegradation reaction of $\mathrm{MB} / \mathrm{RhB}$ with KSbPON shows pseudo-first-order kinetics. The production of $\cdot \mathrm{OH}$ in the presence of KSbPON during the photoreaction is confirmed by fluorescence technique using terephthalic acid (TA) as the probe.

Acknowledgments The authors gratefully acknowledge the UGC (UPE) and DST-FIST, New Delhi, for financial assistance.

\section{References}

[1] C. Xiaobo, S. Shaohua, G. Liejin, S.M. Samuel, Chem. Rev. 110, 6503 (2010)

[2] K. Anna, F.G. Marcos, C. Gerardo, Chem. Rev. 112, 1555 (2012)

[3] A. Fujishima, K. Honda, Nature 238, 37 (1972)

[4] N.P. Titus, V.G. Molina, M.A. Banos, J. Gimenez, S. Esplugas, Appl. Catal. B Environ. 47, 219 (2004)

[5] C. Karunakaran, R. Dhanalakshmi, Sol. Energy Mater. Sol. Cells 92, 1315 (2008)

[6] D. Chatterjee, S. Dasgupta, J. Photochem. Photobiol. C Photochem. Rev. 6, 186 (2005)

[7] M.R. Hoffman, S.T. Martin, W. Choi, D.W. Bahnemann, Chem. Rev. 95, 69 (1995)

[8] X. Zong, L. Wang, J. Photochem. Photobiol. C Photochem. Rev. 18, 32 (2014)
[9] M. Kazuhiko, D. Kazunari, J. Phys. Chem. C 111, 7851 (2007)

[10] B.D. Mert, B. Yazici, Acta Metall. Sin. (Engl. Lett.) 28, 858 (2015)

[11] B. Tang, G. Jiang, Z. Wei, X. Li, X. Wang, T. Jiang, W. Chen, J. Wan, Acta Metall. Sin. (Engl. Lett.) 27, 124 (2014)

[12] G. Jiang, X. Li, Z. Wei, T. Jiang, X. Du, W. Chen, Acta Metall. Sin. (Engl. Lett.) 28, 460 (2015)

[13] Z. Chen, W. Wang, K. Zhu, Acta Metall. Sin. (Engl. Lett.) 28, 1 (2015)

[14] H. Chen, G.H. Jiang, Y.K. Liu, L. Li, Q. Huang, W.X. Chen, Acta Metall. Sin. (Engl. Lett.) 28, 1156 (2015)

[15] H. Hongwei, H. Ying, L. Zheshuai, K. Lei, Z. Yihe, J. Phys. Chem. C 117, 22986 (2013)

[16] K.M. Parida, N. Sahu, N.R. Biswal, B. Naik, A.C. Pradhan, J. Colloid Interface Sci. 318, 231 (2008)

[17] C.S. Pan, Y.F. Zhu, Environ. Sci. Technol. 44, 5570 (2010)

[18] Z.G. Yi, J.H. Ye, N. Kikugawa, T. Kako, S.X. Ouyang, H. Stuart Williams, H. Yang, J.Y. Cao, W.J. Luo, Z.S. Li, Y. Liu, R.L. Withers, Nat. Mater. 9, 559 (2010)

[19] E. Wang, M. Greenblatt, Chem. Mater. 3, 542 (1991)

[20] A. Lachgar, S. Deniard-Courant, Y. Piffard, J. Solid State Chem. 63, 409 (1986)

[21] Y. Piffard, S. Oyetola, S. Courant, A. Lachgar, J. Solid State Chem. 60, 209 (1985)

[22] Y. Piffard, A. Lachgar, M. Toumoux, J. Solid State Chem. 58, 253 (1985)

[23] Y. Piffard, A. Lachgar, M. Tournoux, Mater. Res. Bull. 21, 1231 (1986)

[24] Y. Piffard, A. Lachgar, M. Tournoux, Mater. Res. Bull. 20, 715 (1985)

[25] V. Radha, K. Ramaswamy, G. Ravi, N.R. Munirathnam, M. Vithal, Z. Anorg. Allg. Chem. 641, 935 (2015)

[26] D. Mitoraj, H. Kisch, Angew. Chem. Int. Ed. 47, 9975 (2008)

[27] G. Li, N. Yang, W. Wang, W.J. Zhang, J. Phys. Chem. C 113, 14829 (2009)

[28] S. Sakthivel, M. Janczarek, H. Kisch, J. Phys. Chem. B 108, 19384 (2004)

[29] Q.Y. Li, B. Yue, H. Iwai, T. Kako, J.H. Ye, J. Phys. Chem. C 114, 4100 (2010)

[30] A.I. Vogel, Textbook of Quantitative Chemical Analysis (Longman Group Ltd, Harlow, 1989)

[31] E. Husson, F. Genet, A. Lachgar, Y. Piffard, J. Solid State Chem. 75, 305 (1988)

[32] G. Ravi, S. Mansouri, S. Palla, M. Vithal, Indian J. Chem. 54A, 20 (2015)

[33] S. Palla, R. Guje, G. Ravi, R. Velchuri, M. Vithal, Acta Metall. Sin. (Engl. Lett.) 28, 216 (2015)

[34] N. Talebian, M.R. Nilforoushan, Thin Solid Films 518, 2210 (2012)

[35] R. Marschall, J. Soldat, M. Wark, Photochem. Photobiol. Sci. 12, 671 (2013) 